\title{
Prediction of the density-pressure-temperature behavior of the 5CB Liquid Crystal, at the Isotropic-Nematic phase transition
}

\section{Predicción del comportamiento densidad-presión-temperatura del cristal líquido $5 \mathrm{CB}$, en la transición de fase isotrópica-nemática}

DOI: $10.46932 /$ sfjdv2n3-002

Received in: May 1st, 2021

Accepted in: Jun 30th, 2021

Luis Eduardo Bañuelos-García

Unidad Académica de Ingeniería Eléctrica, Universidad Autónoma de Zacatecas, Zacatecas, México 98600

\section{Luis Octavio Solís Sánchez}

Unidad Académica de Ingeniería Eléctrica, Universidad Autónoma de Zacatecas, Zacatecas, México 98600

\section{Luis Humberto Mendoza Huizar}

Academic Area of Chemistry. Autonomous University of Hidalgo State. Mineral de la Reforma, Hidalgo, Mexico

\section{Francisco Javier Martínez Ruíz}

Unidad Académica de Ingeniería Eléctrica, Universidad Autónoma de Zacatecas, Zacatecas, México 98600

\section{José Manuel Cervantes Viramontes}

Unidad Académica de Ingeniería Eléctrica, Universidad Autónoma de Zacatecas, Zacatecas, México 98600

\section{Eduardo García Sánchez}

Unidad Académica de Ingeniería Eléctrica, Universidad Autónoma de Zacatecas, Zacatecas, México 98600

E-mail: eduardogarciasanchez@gmail.com

\begin{abstract}
In this work, we have analyzed of density-pressure-temperature behavior at the Isotropic-Nematic phase transition of the 5CB liquid crystal at $1 \mathrm{~atm}$ by using a first-order perturbation theory for Convex Peg HERSW model. We predicted quantitatively the experimental behavior in this region of the diagram phase.
\end{abstract}

Keywords: 5CB, isotropic-nematic transition, Convex Peg HERSW model

\section{RESUMEN}

En este trabajo hemos analizado el comportamiento densidad-presión-temperatura en la transición de fase Isotrópica-Nemática del cristal líquido 5CB a 1 atm utilizando una teoría de perturbación de primer orden para el modelo Convex Peg HERSW. Predecimos cuantitativamente el comportamiento experimental en esta región del diagrama de fase. 
Palabras clave: 5CB, transición isotrópica-nemática, modelo Convex Peg HERSW

\section{INTRODUCCION}

Researchers are able to study or predict the qualitative and/or quantitative experimental behavior of some phenomena through theoretical models; and these models allow us to understand and to do inferences about them (Allen \& Tildesley, 1991). Thus, the acceptation of a theoretical model is given after the model is tested, thus, it is necessary to evaluate its prediction capacity. The validation of a theoretical model requires a comparison of the predicted results with those obtained from the experiment (Cervantes et al., 2017). However, in some cases, when a new theoretical model is proposed, in most of the cases, it is only able to describe the qualitative behavior and, partially, the quantitative behavior of the phenomenon. Likely it is due to the either the approximations employed in the theoretical model or a missing academic training (Jaik, 2013). In this sense, the lack of methodologies to develop new theoretical models suggest that each model is highly specific and the criteria employed to develop them are particular.

Specifically, in the field of the prediction of phase transition diagrams of Liquid Crystals (LC), it is possible to develop a procedure with a detailed description of the stages involved during their prediction (Allen, 1995). Thus, in this work, we describe a detailed methodology to develop, verify and validate the prediction capacity of a theoretical model to reproduce the experimental behavior of a Liquid Crystal. We select an LC, which presents intermediate states of the matter (mesophases) between the Isotropic liquids (gases and liquids) and the crystalline solids. Also, it is required the existence of experimental data of the density-pressure-temperature behavior in the Isotropic-Nematic (I-N) phase transition of the LC chosen (Van Hecke, 2001; Deschamp, et al., 2008), which allow us, through a fitting process to generate the equations of state, which are necessaries to do the validation of the theoretical model.

In the present work, the validation process of a theoretical model will be carried out, implementing the MEPPS methodology, consisting of the stages of Concept or pre-production, analysis, design, development, implementation, validation and verification, elaboration of complementary material and conclusions and perspectives (Cervantes et al., 2017), to predict the density-pressure-temperature experimental behavior of the 4-n-pentyl-4-cyano-biphenyl (5CB) LC in the Isotropic-Nematic (I-N) phase transition at $1 \mathrm{~atm}$, which has not been fully predicted.

\section{CONCEPT OR PRE-PRODUCTION}

$5 \mathrm{CB}$ is one of the most studied Liquid Crystals (LC's), because it has a Nematic (N) phase at temperatures between 297 and $308.3 \mathrm{~K}$, has a low melting temperature, is chemically stable in a wide range of temperature, is characterized by a large birefringence, dielectric anisotropy and small viscosity. 
Due to these characteristics, 5CB has been used mainly in electronic displays of watches, mobile phones or computers (Gray et al., 1973). It can also be used as a lubricant because reduce friction between surfaces (Cognard, 1989), since the low friction coefficients are maintained in the $\mathrm{N}$ phase, even when the temperature increases and the I-N phase transition is exceeded, this occurs because the I-N phase transition depends on the pressure and variations in the density. For this reason, that it is necessary to have a broad knowledge from the theoretical and experimental point of view, of the density-pressure-temperature behavior in the I-N phase transition of the $5 \mathrm{CB}$ to $1 \mathrm{~atm}$.

\section{ANALYSIS}

At this stage, experimental data are consulted in the literature and are used in the validation process of the theoretical model. The density-temperature experimental behavior in the I-N phase transition of 5CB (Deschamp, et al., 2008) is given by the following expression:

$$
\rho(T) / K g \cdot m^{-3}=a_{0}+a_{1}\left[\left(T-T_{I N}\right) /^{\circ} K\right]
$$

In this expression, $a_{0}$ y $a_{1}$ are adjustable parameters (Table I), $\rho$ is the density, $T$ is the temperature and $T_{I N}$ is the value of the temperature at the I-N phase transition,

Table I. Fitting parameters $a_{0}$ y $a_{1}$ for density-temperature behavior in the I-N phase transition for 5CB.

\begin{tabular}{rcc}
\hline \hline Phase & $a_{0}$ & $a_{1}$ \\
\hline Isotropic & 1006.44 & -0.7762 \\
Nematic & 1010.48 & -0.9230 \\
\hline \hline
\end{tabular}

The corresponding expression that describes the pressure-temperature experimental behavior in the I-N phase transition from 5CB (Van Hecke, 2001) is:

$$
T / K=a_{2}+a_{3}(P / \text { bar })+a_{4}(P / \text { bar })^{2}
$$

where $a_{2}, a_{3}$ and $a_{4}$ are fitting parameter (Table II), and $P$ is the pressure,

Table II. Fitting parameters $a_{2}, a_{3}$ and $a_{4}$ for pressure-temperature I-N phase transition for 5CB.

\begin{tabular}{ccc}
\hline \hline$a_{2}$ & $a_{3} / 10^{-2}$ & $a_{4} / 10^{-6}$ \\
\hline 308.28 & 4.24 & -3.20 \\
\hline \hline
\end{tabular}


When making the sensitivity analysis of non-formal, conceptual or theoretical models, it was found that in general, the theoretical models that analyze the experimental behavior in the I-N phase transition present three different types of phase diagrams (García-Sánchez et al., 2000). The phase diagram type 1, presents a Vapor-Liquid coexistence (V-L) that ends in a critical point $T_{c}$, a Vapor-Liquid-Nematic triple point (V-L-N) below which the $\mathrm{N}$ phase coexists with the phase of Vapor (V). Between the triple point and the critical point, the $\mathrm{N}$ phase coexists with the Liquid phase (L) and above the critical point with a supercritical Isotropic Fluid. In the type 2 phase diagram, the V-L coexistence is absent, this, because it presents a marked widening of the region between phases I and $\mathrm{N}$, therefore, the critical point $\mathrm{V}-\mathrm{L}$ and the V-L-N triple point do not appear. Finally, in the type 3 phase diagram, the attractive interactions stabilize the I phase with respect to the $\mathrm{N}$ phase, resulting a separation of the phase transition at high densities, the V-L coexistence ends at the critical point. On the other hand, the destabilization of phase $\mathrm{N}$ is such that the I-N phase transition is not connected to the V-L coexistence, therefore, the V-L-N triple point is not observed.

In order to identify the range of validity of the theoretical model, it is necessary to consider the limitations that may have the theoretical model, for example the existence of ordered phases, such as a Liquid-Vapor-Smectic triple point (L-V-Sm) or Liquid-Vapor-Crystalline Solid triple point, which can occur, since the triple point, for the I-N phase transition occurs at a pressure equal to zero, which would be very close to the triple points $\mathrm{L}-\mathrm{V}-\mathrm{Sm}$ and $\mathrm{L}-\mathrm{V}-\mathrm{K}$. However, here it is important to mention that $5 \mathrm{CB}$ experimentally does not present these behaviors. Moreover, 5CB shows a phase diagram type 1 . Thus, the field of action of the theoretical model proposed in this work will focus on analyzed the region of the diagram type 1 where the I-N phase transition is observed.

\section{DESIGN}

The non-formal model, utilized in this paper, is expressed by a first-order Baker-Henderson type perturbation theory for the HERSW Convex Peg model (García-Sánchez et al., 2000), in which, the $N$ molecules in a volume $V$ at a temperature $T$ are characterized by a hard uniaxial ellipsoidal core (HER) surrounded by a square well (SW) which is close to the edge of the hard core at the end of its major axis. The expression for the Helmholtz free-energy is given by: 


$$
\begin{aligned}
& a=\frac{A-A^{i d}}{N k_{B} T}=\int f(\Omega) \ln [4 \pi f(\Omega)] d \Omega+\frac{<V_{\text {excl }}^{H C}\left(\Omega_{1}, \Omega_{2}\right)>_{\Omega_{1}, \Omega_{2}}}{8 V_{m}}\left[\frac{4 \eta-3 \eta^{2}}{(1-\eta)^{2}}\right]-4 \eta\left(e^{\frac{1}{T^{*}}}-1\right) \\
& {\left[\left(\frac{b_{\lambda}}{b}\right)^{2} \frac{<V_{\text {excl }}^{\text {atr }}\left(\Omega_{1}, \Omega_{2}\right)>_{\Omega_{1}, \Omega_{2}}}{<V_{\text {excl }}^{H C}\left(\Omega_{1}, \Omega_{2}\right)>_{\Omega_{1}, \Omega_{2}}}-1\right]\left(\frac{<V_{\text {excl }}^{H C}\left(\Omega_{1}, \Omega_{2}\right)>_{\Omega_{1}, \Omega_{2}}}{8 V_{m}}\right)}
\end{aligned}
$$

Where $\frac{V_{m}^{a t r}}{V_{m}^{H C}}=\left(\frac{b_{\lambda}}{b}\right)^{2}, f(\Omega)$ is the one particle orientational distribution function for a solid angle $\Omega, V_{\text {excl }}^{H C}\left(r_{12}, \Omega_{1}, \Omega_{2}\right)$ is the excluded volume generated by the interaction between hard cores of two particles with orientation $\Omega_{1}$ and $\Omega_{2}, V_{\text {excl }}^{\text {atr }}\left(r_{12}, \Omega_{1}, \Omega_{2}\right)$, is the excluded volume generated by the interaction between the attractive core of a particle with an orientation $\Omega_{2}$, and the attractive core of another particle oriented around $\Omega_{1}$. The general expression is:

$$
\frac{<V_{\text {excl }}\left(\Omega_{1}, \Omega_{2}\right)>_{\Omega_{1}, \Omega_{2}}}{8 V_{m}}=d_{0}(k)-16 \pi^{2} \sum_{n=1}^{4} d_{n}(k)\left[\int_{0}^{1} P_{n}(x) f(x) d x\right]^{2}
$$

Where, for the case of the excluded volume of a hard $\operatorname{core}, x=\cos \theta, d x=-\operatorname{sen} \theta d \theta$,

\begin{tabular}{|c|c|}
\hline & $d_{n}(k)$ \\
\hline$d_{2}(k)$ & $\begin{array}{l}1.0404224126634527-0.2124822065050437911 k+0.200376367800779497 k^{2}- \\
0.0452960284393375545 k^{3}+0.00574306142400010699 k^{4}- \\
0.000379115304850269341 k^{5}+0.0000101212234726092131 k^{6} \\
0.0179333444346647663-0.181480728982367889 k+0.19209470067370038 k^{2}- \\
0.047788875213772485 k^{3}+0.00632342440673977534 k^{4}- \\
0.00042675267423366616 k^{5}+0.0000101212234726092131 k^{6}\end{array}$ \\
\hline$d_{4}(k)$ & $\begin{array}{l}0.0194078111400190493-0.0239566112411885301 k+0.00203625455229150542 k^{2} \\
+0.00598992119559058089 k^{3}-0.00180995207304058407 k^{4} \quad+0.00024059689272044018 k^{5}- \\
0.0000222110948667727814 k^{6} \\
+0.00000100621182481881099 k^{7}\end{array}$ \\
\hline$d_{6}(k)$ & $\begin{array}{l}-0.010292896516519108+0.0245974193467313462 k-0.022282083144730383 k^{2} \\
+0.00958219741464688645 k^{3}-0.00210026578555277954 k^{4} \\
+0.000276712894861745617 k^{5}-0.000022211094866727814 k^{6} \\
+0.00000100621182481881099 k^{7}-0.0000000197936446851484646 k^{8}\end{array}$ \\
\hline$d_{8}(k)$ & $\begin{array}{l}0.00340145568249058971-0.0103737138719856858 k+0.0128603133989782225 k^{2} \\
-0.00834614249029559651 k^{3}+0.00306427700897882848 k^{4}- \\
0.00065850083307786612 k^{5}+0.0000871812524289356005 k^{6}- \\
0.00000703189845731186569 k^{7}+0.000000317576022608253305 k^{8}- \\
0.0000000061611627577897798 k^{9}\end{array}$ \\
\hline
\end{tabular}
$P_{n}(\cos \theta)=P_{n}(x)$ are the even Legendre polynomials, and finally, the even values for $d_{n}(k)$ are presents in Table III. 
The state, input, intern, and output parameters and variables of the non-formal model are described in Table IV, as well as their respective restrictions.

Table IV. Non-formal model parameters and variables with their characteristics.

\begin{tabular}{|c|c|c|c|c|}
\hline Parameter & Name & Type & Constraints & Units \\
\hline$A$ & Helmholtz free energy & & & $J$ \\
\hline$A^{i d}$ & Ideal Helmholtz free energy & & & $J$ \\
\hline$N$ & Number of molecules & & $(0, \infty)$ & \\
\hline$k_{B}$ & Boltzmann constant & constant & $1.38064852 \times 10^{-23}$ & $J / K$ \\
\hline$T^{*}=k_{B} T / \varepsilon$ & Reduced temperature & input & $(0, \infty)$ & \\
\hline$T$ & Temperature & state & $(0, \infty)$ & $K$ \\
\hline$P$ & Pressure & state & {$[0, \infty)$} & Bar \\
\hline$P_{I}^{*}$ & Reduced Pressure I & output & {$[0, \infty)$} & \\
\hline$P_{N}^{*}$ & Reduced Pressure N & output & {$[0, \infty)$} & \\
\hline$\rho$ & Density & state & & \\
\hline$\mu$ & Chemical potential & state & & \\
\hline$\mu_{I}^{*}$ & Chemical potential I & output & & \\
\hline$\mu_{N}^{*}$ & Chemical potential $\mathrm{N}$ & output & & \\
\hline$\varepsilon$ & Depth of square well & & $(0, \infty)$ & $J$ \\
\hline$\eta=N V_{m} / V$ & Atomic packing factor & & {$[0,1]$} & \\
\hline V & System volume & state & $(0, \infty)$ & $m^{3}$ \\
\hline$V_{m}$ & Molecular volume & & $(0, \infty)$ & $m^{3}$ \\
\hline$V_{m}^{H C}$ & Hard core molecular volume & & $(0, \infty)$ & $m^{3}$ \\
\hline$\eta_{I}$ & Atomic packing factor I & output & {$[0,1]$} & \\
\hline$\eta_{N}$ & Atomic packing factor $\mathrm{N}$ & output & {$[0,1]$} & \\
\hline$V_{m}^{a t r}$ & Attractive core molecular volume & & $V_{m}^{a t r}>V_{m}^{H C}$ & $m^{3}$ \\
\hline$k=a_{p} / b$ & Hard ellipsoid principal axis to semi-axis ratio & input & {$[3,10]$} & \\
\hline$b$ у $c$ & Hard ellipsoid semi-axes & & $b=c$ & \\
\hline$a_{p}$ & Hard ellipsoid principal axis & & $a_{p}>b$ & \\
\hline$k^{\prime}=a_{p} / b_{\lambda}$ & Attractive ellipsoid principal axis to semi-axis ratio & input & {$[3,10]$} & \\
\hline$b_{\lambda}$ y $c_{\lambda}$ & Attractive ellipsoid semi-axes & & $b_{\lambda}=c_{\lambda}$ & \\
\hline$b_{\lambda} / b$ & $\begin{array}{l}\text { Hard core semi-axis to attractive core semi-axis } \\
\text { ratio }\end{array}$ & input & $\frac{b_{\lambda}}{b}=\sqrt{\frac{V_{m}^{a t r}}{V_{m}^{H C}}}$ & \\
\hline$\alpha$ & Variational parameter & intern & & \\
\hline
\end{tabular}

The pressure and chemical potential reduced can be determined by: 


$$
P^{*}=\frac{P V_{m}^{H C}}{k_{B} T}=\eta\left[1+\eta\left(\frac{\partial\left(A-A^{\text {ideal }}\right)}{\partial \eta}\right)\right]
$$

And

$$
\mu^{*}=\frac{\mu}{k_{B} T}=\ln (\eta)+\left(\frac{A-A^{\text {ideal }}}{N k_{B} T}\right)+\eta\left(\frac{\partial\left(A-A^{\text {ideal }}\right)}{\partial \eta}\right)
$$

In order to obtain the non-formal model values that would predict the density-pressure-temperature behavior in the I-N phase transition of $\mathrm{LC} 5 \mathrm{CB}$, it is necessary to perform a minimization process of the functional of free Helmholtz energy, for which it is used the test function method, since the free Helmholtz energy depends on de $f(\Omega)$. This process consists of (see figure 1):

- Select and substitute the input parameters $T^{*}, b_{\lambda} / b, k$ y $k^{\prime}$ in the expression of free Helmholtz energy.

- The free Helmholtz energy in the $\mathrm{N}$ phase, is obtained by replacing and evaluating the Onsager (Onsager, 1949) test function $f(\Omega)=[\alpha \cosh (\alpha \cos \theta) / 4 \pi \operatorname{senh} \alpha]$ in Eq. (3), and performing the respective operations. After that, it is followed the minimization process for internal parameter $\alpha$, which consists in minimizing the free energy over a wide atomic packing factors range of values. The minimized values for the internal parameter are adjusted for a polynomial in function of the atomic packing factor and then, the values are replaced again in the free energy functional obtaining the minimized free energy for the Nematic phase.

- The Helmholtz free energy in I phase, is obtained by replacing and evaluating $f(\Omega)=1 / 4 \pi$ in Eq. (3) and performing the respective operations.

- The pressure and chemical potential expressions for each phase ( $\mathrm{I}$ and $\mathrm{N}$ ), are obtained using Eqs. (5) and (6). By means of these expressions the atomic packing factor values are found, for the $\mathrm{N}$ phase $\eta_{N}$ as well as for I phase $\eta_{I}$, accomplishing the equilibrium condition, which implies that chemical potential $\left(\mu_{N}^{*}=\mu_{I}^{*}\right)$ and pressure $\left(p_{N}^{*}=p_{I}^{*}\right)$ are equal respectively.

Figure 1. Flow diagram for the Helmholtz free energy minimization process. 


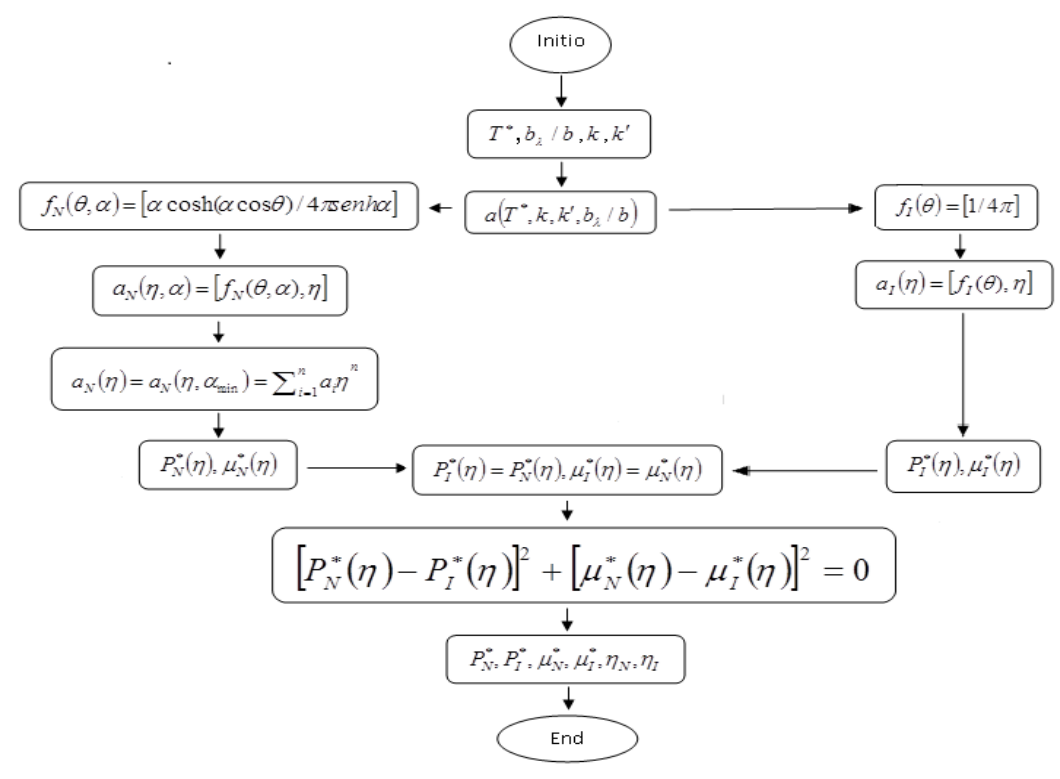

\section{DEVELOPMENT}

To carry out the codification it was used Mathematica 4.0 software, and the calculations to determine the theoretical data were obtained in a Workstation DELL Precision T7500 Xeon $2.26 \mathrm{GHz} 48$ Gb Ram 2 Tb with Windows XP. Additionally, by revising literature, in Table V, are presented several external values of variables and parameters from the theoretical model that cannot be directly calculated, and that are crucial to carry out the experimental data comparison.

Table V. Theoretical parameters for 5CB (García-Sánchez et al, 2015).

\begin{tabular}{ccccc}
\hline \hline$V_{m}^{H C} / \AA^{3}$ & $V_{m}^{a t r} / \AA^{3}$ & $k$ & $k^{\prime}$ & $b_{\lambda} / b$ \\
\hline 80.19 & 257.60 & 3.7 & 2.065 & 1.792 \\
80.19 & 257.60 & 3.8 & 2.121 & 1.792 \\
\hline \hline
\end{tabular}

\section{IMPLEMENTATION}

For the implementation stage, initially the program code is generated, based on the theoretical model by following a flow diagram. Nevertheless, it is important to notice that during the process syntax mistakes, non-valid processes, etc. are made, although they are detected in the validation and verification stage, when applying the MEPPS methodology (Cervantes et al., 2017), the process is returned of the implementation stage to carry out the debugging of the program and correct the errors detected, obtaining the final program of the theoretical model.

\section{VALIDATION AND VERIFICATION}


Verification of the non-formal model. The verification process was carried out by the assigned reviewers, by the molecular physics journal editorial committee, which gave as a result the article publication in which the non-formal model is described (García-Sánchez et al., 2000).

Validation of the non-formal model. The process is composed by three fundamental stages, which are:

A. Experts Validation. The verification process was carried out by the molecular physics journal reviewers, finding that this model is representative of the real system, since it describes qualitatively the experimental behavior in the phase I-N transition (García-Sánchez et al., 2000).

B. Measurements in the real system. The validation process was carried out by finding that the nonformal model may partially describe, and in a quantitative way, the experimental behavior of the I-N transition for several LC's (García-Sánchez et al., 2015)

C. Theoretical results. This part of the validation process cannot be carried out, since it is required to have simulation data that uses exactly the same non-formal model, and as far as we know, they are not reported in the literature.

Verification of the formal model. This process was initially carried out by verifying the algorithms that conform computational program that accomplish the design stage defined requirements, and then, were properly implemented. It was carried out on the one hand by running the computational program and verifying that there is not any syntax mistake, on the other hand by removing the sentence “;" at every code line ending and then running the program, so that the operation or programed sentence is analytically deployed from the non-formal model in every code line. When syntax mistakes were detected, the process returned to the implementation stage, and then it was made the correction and depuration of the simulation program.

Validation of the Formal Model. To carry out the validation process, they were made test runs using:

- Valid input parameters or variables that accomplish the defined restrictions for the non-formal model.

- Input parameters or variables that did not accomplish the defined restrictions for the non-formal model, in order to detect mistakes, besides, it would delimitate and validate the field of action of the formal model.

In the cases in which there were found mistakes, it was returned to the implementation stage to identify and rectify them.

Experiment design. It was found:

A) The start time takes from 5 to 10 seconds. 
B) Cycles cannot be quantified.

C) The required ratio between the computation time and the margin of error for the equilibrium condition to be accomplished $\Delta E=\left[P_{N}^{*}\left(\eta_{N}\right)-P_{I}^{*}\left(\eta_{I}\right)\right]^{2}+\left[\mu_{N}^{*}\left(\eta_{N}\right)-\mu_{I}^{*}\left(\eta_{I}\right)\right]^{2}$ can be observed in Table VI, where $\mathrm{t}$ represents the time taken to find a simulation data, which is approximately $50 \mathrm{~s}$ with $\Delta E \approx 10^{-20}$.

Table VI. Real time vs. equilibrium condition.

\begin{tabular}{cc}
\hline \hline$t / s$ & $\Delta E$ \\
\hline 17.17 & $2.66 \times 10^{-7}$ \\
29.51 & $3.60 \times 10^{-10}$ \\
39.05 & $2.14 \times 10^{-14}$ \\
48.98 & $1.68 \times 10^{-20}$ \\
\hline \hline
\end{tabular}

When delimitating the field of action of the non-formal model, it was considered only the analysis of the phase diagram part of interest, specifically, the experimental behavior in the I-N phase transition. Such behavior is determined by initially taking large values of $T^{*}$ and setting the input parameters in which at the end of the process positive values of $P^{*}$ will be obtained, subsequently, smaller values of $T^{*}$ will be used, until the value of $P^{*}$ would be almost zero (with a margin of error of $10^{-4}$ ), this value of $T^{*}$ corresponds to the triple point of the I-N phase transition.

Optimization. In order to optimizing the process, we determinate graphically the optimal approximate values for the packing factor that fulfill the equilibrium condition for the I-N phase transition. This permits notably to decrease the computation time that it takes to find a point in the phase diagram. The process consists in assigning a range of values to the packing factors $\mathrm{I}$ and $\mathrm{N}$ to evaluate the pressure and chemical potential for each phase, after that, the next step is visualizing graphically those functions, until determining which values for the packing factor, when evaluated, permit to find intersections, as it can be observed in figure 2, these approximate values are used to start the minimization process.

Figure 2. One point in the I-N Phase transition obtained with optimization process.

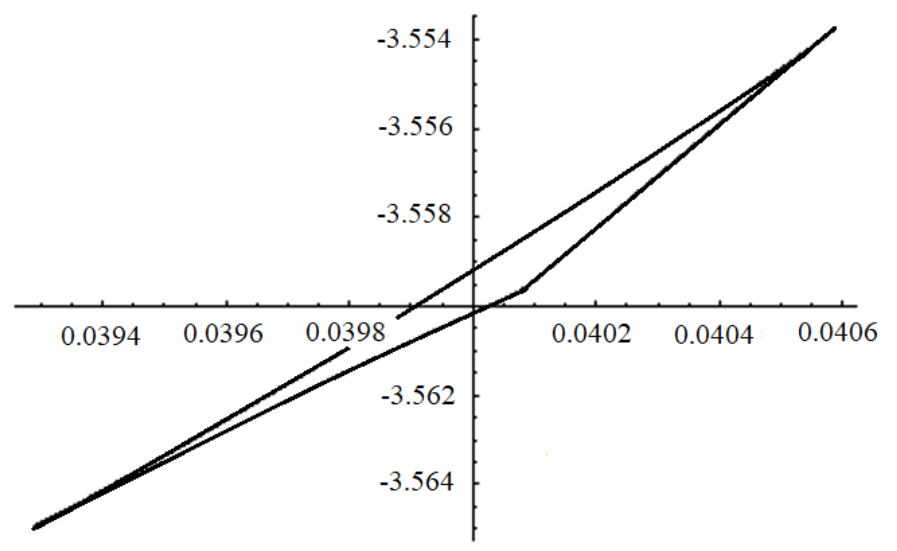


Operational verification or Results verification. In order to carry out the verification, the next activities must be done:

A. Calibration. The calibration process can be carried out, when having reported data from the literature, obtained with the same theoretical model, which in this case is not available.

B. Data Generation and documentation of the model. In Tables VII and VIII the theoretical model obtained data are shown for $k=3.7$ and $k=3.8$.

Table VII. Obtained parameters using the theoretical model for the I-N phase transition when $k=3.7$ and $k^{\prime}=2.065$.

\begin{tabular}{ccccc}
\hline \hline$T^{*}$ & $\eta_{I}$ & $\eta_{N}$ & $P^{*}$ & $\mu^{*}$ \\
\hline 1 & 0.482182 & 0.506945 & 4.89531 & 8.39747 \\
0.9 & 0.479748 & 0.50641 & 4.12149 & 5.47045 \\
0.8 & 0.476004 & 0.50589 & 3.03895 & 1.34955 \\
0.7 & 0.469668 & 0.506223 & 1.44705 & -4.78513 \\
0.62 & 0.464342 & 0.507584 & 0.344606 & -9.08761 \\
0.64 & 0.462979 & 0.508069 & 0.0912092 & -10.0899 \\
0.637 & 0.462465 & 0.508237 & 0.0102897 & -10.3996 \\
0.636 & 0.462315 & 0.508294 & 0 & -10.504 \\
\hline \hline
\end{tabular}

Table VIII. Obtained parameters using the theoretical model for the I-N phase transition when $k=3.8$ and $k^{\prime}=2.121$

\begin{tabular}{ccccc}
\hline \hline$T^{*}$ & $\eta_{I}$ & $\eta_{N}$ & $P^{*}$ & $\mu^{*}$ \\
\hline 1 & 0.474669 & 0.498274 & 4.57235 & 7.80319 \\
0.9 & 0.473171 & 0.499193 & 3.87257 & 5.02098 \\
0.8 & 0.470837 & 0.50076 & 2.8867 & 1.08989 \\
0.7 & 0.466579 & 0.504012 & 1.40953 & -4.82001 \\
0.64 & 0.461595 & 0.508135 & 0.121189 & -9.9894 \\
0.636 & 0.461127 & 0.508499 & 0.0198946 & -10.3964 \\
0.635 & 0.461006 & 0.508592 & 0 & -10.4996 \\
\hline \hline
\end{tabular}

C. Data simulation verification. In this stage the verification process was performed for data shown in Tables VII and VIII, finding they are reproducible and that variations are not significant.

Data processing. To determine the prediction capacity for the theoretical model, it is necessary to calculate the square well value depth $\varepsilon=\varepsilon_{I N}$ (see Table IX) using $T^{*}=\frac{K_{B} T}{\varepsilon}$ in the triple point ( $T^{*} \equiv T_{T r}^{*} \equiv T_{I N}^{*}$ ) with the experimental value $308.65^{\circ} \mathrm{K}$ for $5 \mathrm{CB}$ at this point, setting this value, the other temperature values would be determined by $T=T^{*} \varepsilon_{I N} / K_{B}$. To calculate pressure, it is used 
$P=P_{i}+\varepsilon T^{*} P^{*} / V_{m}=P_{i}+\varepsilon_{I N} T^{*} P^{*} / V_{m}{ }^{H C}$ (where $\left.P_{i}=1 \mathrm{~atm}\right)$ and to determine density it is used $\rho=\frac{M_{r} \eta}{N_{A} V_{m}}$, where $N_{A}$ is Avogadro constant $\left(6.022 \times 10^{23}\right.$ molecules $\left./ \mathrm{mol}\right)$ and $M_{r}$ is the molar mass or relative molecular mass $(249.342 \mathrm{~g} / \mathrm{mol}$ for $5 \mathrm{CB})$. To determine density in phase $\mathrm{I} \rho_{I}=\frac{M_{r} \eta_{I}}{N_{A} V_{m}}$ and for N $\rho_{N}=\frac{M_{r} \eta_{N}}{N_{A} V_{m}}$ are used.

Table IX. 5CB parameters and variables for the I-N phase transition.

\begin{tabular}{ccccc}
\hline \hline$k$ & $k^{\prime}$ & $T_{I N}{ }^{*}$ & $\varepsilon_{I N} / J$ & $V_{m}^{H C} / \AA^{3}$ \\
\hline 3.7 & 2.065 & 0.636 & $6.697 \times 10^{-21}$ & 80.19 \\
3.8 & 2.121 & 0.635 & $6.707 \times 10^{-21}$ & 80.19 \\
\hline \hline
\end{tabular}

Operational verification or Results verification. Respecting to pressure-temperature behavior in the phase diagram region, described by phase transition I-N, the theoretical model predicts quantitatively this behavior for 5CB with $k=3.7$ and $k^{\prime}=2.065$ (see figure 3(a)) and for $k=3.8$ and $k^{\prime}=2.121$ (see figure 3(b)). Respecting to density-temperature behavior, it can be observed that in Isotropic and Nematic behavior, in figure 4(a) for $k=3.7$ and $k^{\prime}=2.065$, and figure 4(b) for $k=3.8$ and $k^{\prime}=2.121$, the theoretical model cannot predict quantitatively this experimental behavior.

Figure 3. Pressure-temperature behavior for the theoretical model (asterisk line) and experimental data (solid line) in the I-N phase transition for 5CB at $1 \mathrm{~atm}$, with (a) $k=3.7$ and $k^{\prime}=2.065$, and (b) $k=3.8$ and $k^{\prime}=2.121$.

Figure 4. Density-Temperature plot for the theoretical model (asterisk line) and experimental data (solid line) in the I-N phase transition for 5CB at $1 \mathrm{~atm}$, in the Isotropic and Nematic behavior with (a) $k=3.7$ and $k^{\prime}=2.065$, and (b) $k=3.8$ and $k^{\prime}=2.121$. 
(a)
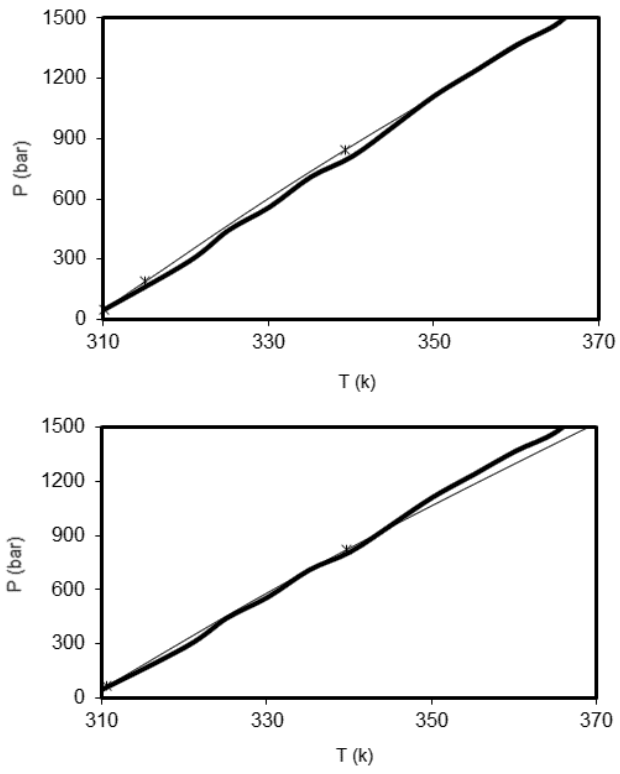

(a)
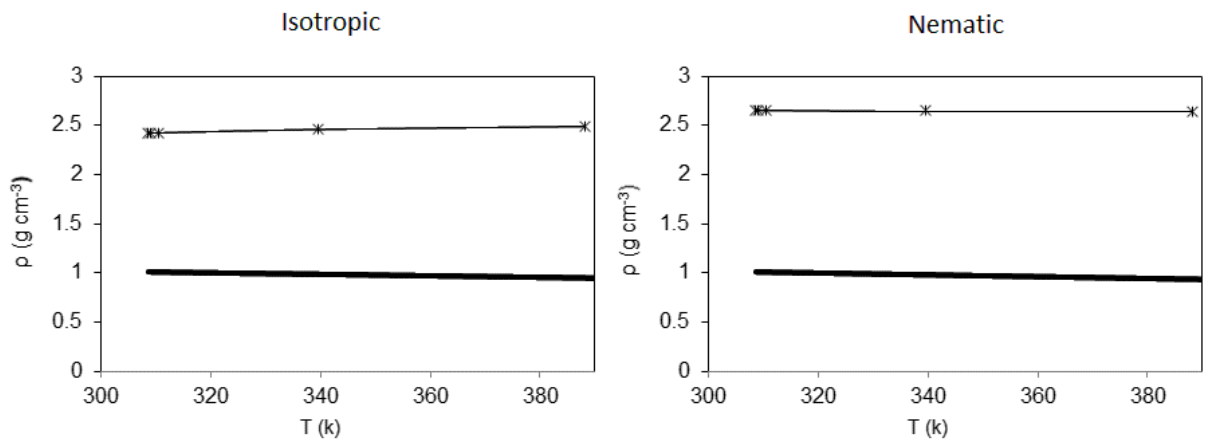

(b)
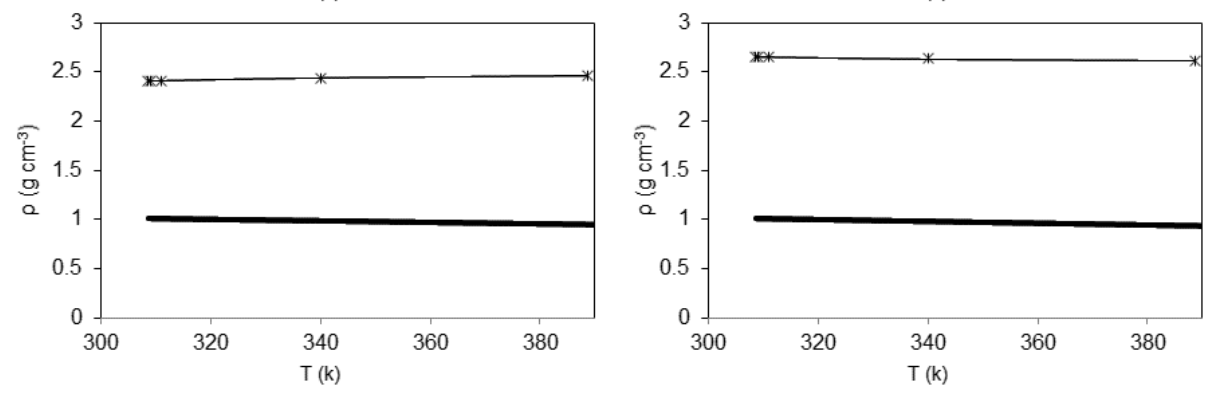

\subsection{SENSITIVITY RESULTS ANALYSIS}

In the previous section it was shown that the theoretical model predicts only partially the pressuredensity-temperature behavior in the phase transition I-N from 5CB at $1 \mathrm{~atm}$. It could had happened since it was not utilized any adjustment parameter in the density experimental value in the transition point I-N. To solve this situation, in this work it is proposed on one hand, that $V_{m}=\gamma_{I N} V_{m}^{H C}=\gamma_{I} V_{m}^{H C}=\gamma_{N} V_{m}^{H C}$, 
where $\gamma_{I N}$ is the adjustment factor in the triple point for phase I-N, $\gamma_{I}$ and $\gamma_{N}$ are the adjustment factors in phase I and $\mathrm{N}$ respectively. And on the other hand, $\varepsilon=\gamma_{I N} \varepsilon_{I N}=\gamma_{I} \varepsilon_{I}=\gamma_{N} \varepsilon_{N}$, where $\varepsilon_{I}$ and $\varepsilon_{N}$ are the potential square well depths in the phases I and $\mathrm{N}$ respectively.

The adjustment parameters that were utilized to predict the pressure-density-temperature experimental behavior in phase transition I-N for $5 \mathrm{CB}$ at 1 atm are presented in Table $\mathrm{X}$

Table X. 5CB parameters or variables required to predict the pressure-density-temperature behavior in the I-N transition phase.

\begin{tabular}{cccccccccc}
\hline \hline$k$ & $k^{\prime}$ & $V_{m}^{H C} / \AA^{3}$ & $T_{I N}^{*}$ & $\gamma_{I N}$ & $\varepsilon_{I N} / J$ & $\gamma_{I}$ & $\varepsilon_{I} / J$ & $\gamma_{N}$ & $\varepsilon_{N} / J$ \\
\hline 3.7 & 2.065 & 80.19 & 0.636 & 1 & $6.697 \times 10^{-21}$ & 2.3718 & $2.8236 \times 10^{-21}$ & 2.5973 & $2.5784 \times 10^{-21}$ \\
3.8 & 2.121 & 80.19 & 0.635 & 1 & $6.707 \times 10^{-21}$ & 2.3659 & $2.8348 \times 10^{-21}$ & 2.5988 & $2.5808 \times 10^{-21}$ \\
\hline \hline
\end{tabular}

Using the parameter values from Table $\mathrm{X}$, the pressure-temperature behavior in the region of the phase diagram, described for phase transition I-N, is not modified, and the results are the same as those presented in figure 3. From the density-temperature experimental behavior, it can be observed that in Isotropic and Nematic behavior, in figure 5(a) for $k=3.7$ and $k^{\prime}=2.065$, and figure 5(b) for $k=3.8$ and $k^{\prime}=2.121$, the theoretical model predicts quantitatively this experimental behavior.

Figure 5. Density-Temperature plot for the theoretical model (asterisk line) and experimental data (solid line) in the I-N phase transition for $5 \mathrm{CB}$ at $1 \mathrm{~atm}$, in the Isotropic and Nematic behavior with (a) $k=3.7$ and $k^{\prime}=2.065$, and (b) $k=3.8$ and $k^{\prime}=2.121$. 
(a)
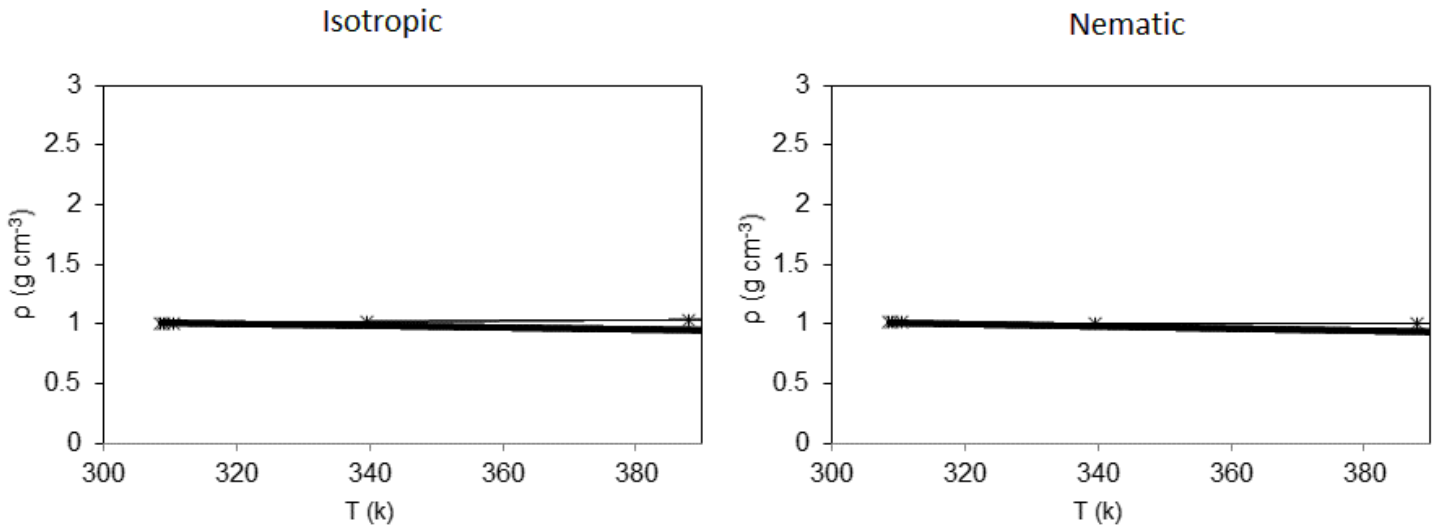

(b)
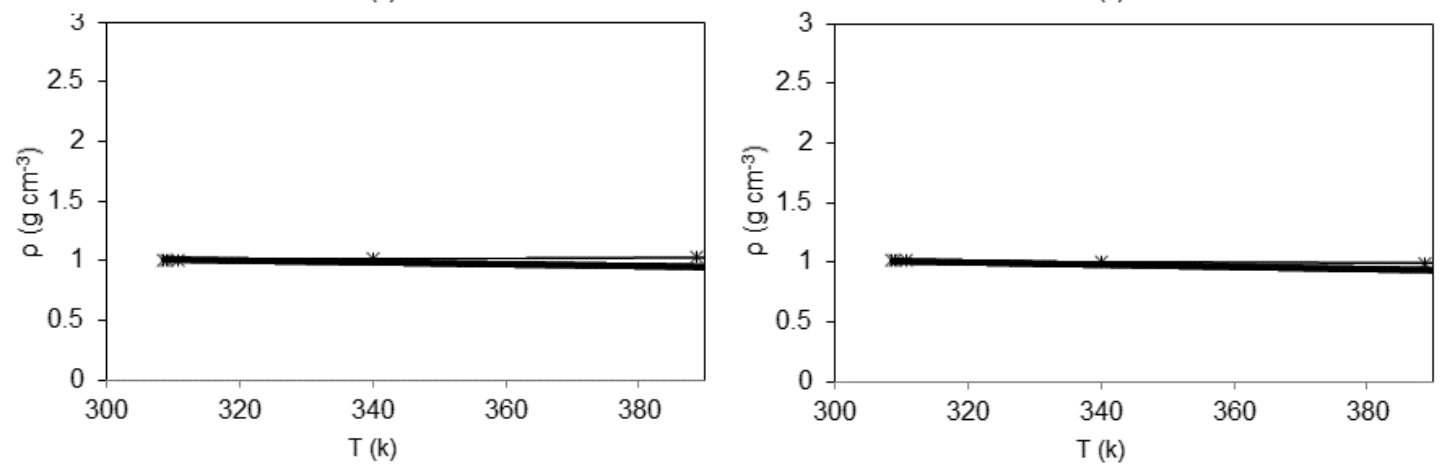

\subsection{RESULTS INTERPRETATION}

It was demonstrated that the density-pressure-temperature experimental behavior in the phase transition I-N, is consistent with the obtained results in this work.

- As in the system it is shown a temperature increase, the molecules start its kinetic energy, which allows the molecules to overcome the short-range interaction, resulting those molecules start to collision causing a pressure increase.

- It is observed that density in N Phase is larger than in I phase, since molecules in N phase are oriented, they can move and be agglomerated more easily, unlike I phase, with the consequence of in $\mathrm{N}$ phase, viscosity is smaller than in I phase.

- As consequence of the equilibrium between both phases, it is found a larger molecule ordering in phase $\mathrm{N}$ with respect to I phase. This promotes an increase of pressure and temperature, which is compensated with small variations in density values for both phases.

- The theoretical model predicts quantitatively the density-pressure-temperature experimental behavior in the phase transition I-N.

Final implementation. Since the theoretical model predicts quantitatively the density-pressuretemperature experimental behavior in the I-N phase transition for $5 \mathrm{CB}$ at $1 \mathrm{~atm}$, the computational program can be utilized to predict this behavior for other Liquid Crystals. 


\section{COMPLEMENTARY MATERIAL ELABORATION}

Respecting to prepare a Technical Manual, the Mathematica 4.0 program has a help button to check the code structure being programming.

\section{CONCLUSIONS AND PERSPECTIVES}

In this work, we have analyzed the experimental density-pressure-temperature behavior of the I$\mathrm{N}$ phase transition of the liquid crystal $5 \mathrm{CB}$ at $1 \mathrm{~atm}$. We predicted quantitative the experimental densitypressure-temperature behavior in this region of the diagram phase with $k=3.7$ and $k^{\prime}=2.065$ and, with $k$ $=3.8$ and $k^{\prime}=2.121$.

Other important factors that might be modified in later studies to improve the theoretical prediction, would be to consider in the Helmholtz free energy expression the following: the model might be described by means of biaxial core, the incorporation of the flexibility and polarity effect, and the consideration of the large range interactions, since it is possible, as the molecule size increases, the contributions to the free energy from these parameters are more important. 


\section{REFERENCES}

Allen, M.P. (1995). Simulations and phase behaviour of Liquid Crystals, in Observation, Prediction and Simulation of Phase Transitions in Complex Fluids. Dordrecht, the Netherlands: Kluwer Academic Publisher.

Allen, M. P., \& Tildesley, D.J. (1991). Computer Simulation of Liquids. Oxford: Clarendon Press.

Cervantes, J. M., García, M. A., Escalera, J. J., Torres, V., \& García-Sánchez, E. (2017). Metodología para el proceso de simulación MEPPS, DIFU100CI@, 10. http://difu100cia.uaz.edu.mx/index.php/difuciencia/article/view/52

Cognard, J. (1989). Lubrication with Liquid Crystal, Chapter 1, in Tribology and the Liquid-Crystalline State, edited by G. Biresaw (Developed from a Symposium Sponsored by the Division of Colloid and Surface Chemistry at the 198th National Meeting of the American Chemical Society). Miami Beach, Florida, USA.

Deschamp, J., Trusler, J. P. M., \& Jackson, G. (2008). Vapor Pressure and Density of Thermotropic Liquid Crystals: MBBA, 5CB and Novel Fluorinated Mesogens. J. Phys. Chem. 112(13), 3918-3926. https://doi.org/10.1021/jp711211w

García-Sánchez, E., Mendoza-Huizar, L. H., Ramírez-García, U., Sustaita, I. A., and Alvarado, F. (2015). Analysis of the Influence of the Molecular Volume to predict experimental pressure-temperature behavior in the Isotropic-Nematic Phase Transition of PAP, 5CB, MBBA and EBBA. Braz. J. Phys. 45, 258-263. http://link.springer.com/article/10.1007/s13538-014-0295-6

García-Sánchez, E., Williamson, D., \& Martínez-Richa, A. (2000). Effects of molecular geometry on liquid crystalline phase behavior: isotropic-nematic transition. Mol. Phys. 98(3), 179-192 (2000). https://doi.org/10.1080/00268970009483281

Gray, G. W., Harrison, K. J., \& Nash, J. A. (1973). New Family of Nematic Liquid Crystals for Displays. Electron. Lett. 9(6), 130-131. https://digital-library.theiet.org/content/journals/10.1049/el_19730096

Jaik, A. (2013). Competencias Investigativas: Una mirada a la Educación Superior. México: Red Durango de Investigadores Educativos ReDIE. https://redie.mx/librosyrevistas/libros/competenciasinvestigativas.pdf

Onsager, L. (1949). The effects of shape on the interaction of colloidal particles, Ann. N. Y. Acad. Sci. 51, 627-659. https://doi.org/10.1111/j.1749-6632.1949.tb27296.x

Van Hecke, G.R. (2001). Phase transition and the effects of pressure in Physical Properties of Liquid Crystals: Nematic. London, United Kingdom: INSPEC. 